\title{
Sir2 function - a two-way street
}

URLs

Calorie restriction (CR) increases lifespan in organisms that are as diverse as yeast and humans; but, as three papers show, the underlying molecular pathways are complex. A gene that was thought to provide the main link between calories and lifespan in yeast turns out to be only part of the story, and in fact has opposite effects on different aspects of longevity.

Yeast longevity can be measured in terms of replicative lifespan - the number of times a cell can divide before dying. The SIR2 gene was initially thought to be essential for CR to increase this type of longevity. One theory is that the depletion of nicotinamide during CR leads to replicative-lifespan extension by derepressing Sir2. To test this, Kaeberlein, Kennedy and colleagues used Saccharomyces cerevisiae cells that were double mutant for SIR2 and a second gene, FOB1 - a combination that suppresses the decrease in replicative lifespan that is caused by SIR2 mutation alone. CR extends lifespan in these cells, but this effect is decreased by nicotinamide addition, showing that this chemical suppresses the lifespan-enhancing effects of CR independently of Sir2.

In a second study, a screen carried out by the same group identified deletions in 10 genes that lead to increased replicative lifespan. Six of these genes encode components of the Tor (target of rapamycin) and
Sch9 pathways, which have been implicated in CR-mediated effects on lifespan in other species. Importantly, these results were unaffected by SIR 2 deletion, again pointing to other CRresponsive mechanisms.

Another blow to SIR 2 as the main lifespan-extending gene in yeast comes from a study by Longo and colleagues, who used a second measure of longevity - the cell's chronological lifespan. Introducing a SIR2 mutation into already long-lived strains further increased longevity, indicating that wild-type SIR2 blocks extreme chronological-lifespan extension. Furthermore, the ability of CR to increase chronological lifespan was enhanced in SIR2 mutants - completely the opposite effect to that seen for replicative lifespan.

Why does SIR2 have such different effects on these two measures of longevity? One possibility is that this gene changes roles at different stages of yeast growth. The intricacies of the situation in a relatively simple model such as yeast point to even greater complexities that are waiting to be unravelled in the regulation of mammalian longevity.

\section{Louisa Flintoft}

\section{ORIGINAL RESEARCH PAPERS}

Kaeberlein, M. et al. Increased life span due to calorie restriction in respiratory-deficient yeast. PLoS Genet. 1, e69 (2005) |Kaeberlein, M. et al. Regulation of yeast replicative life span by TOR and Sch9 in response to nutrients. Science $\mathbf{3 1 0}$ 1193-1196 (2005) | Fabrizio, P. et al. Sir2 blocks extreme life-span extension. Cell 123, 655-667 (2005) 